\title{
Newborn Electrocardiography as a Screening Method for Long-QT Syndrome
}

\author{
Torres $\mathrm{F}^{1}$, Hernández $\mathbf{M}^{1}$, Garcia $\mathrm{J}^{1}$, Marti-Almor $\mathrm{J}^{3}$ and Garcia-Algar $\mathbf{O}^{1,2^{*}}$ \\ ${ }^{1}$ Paediatrics Unit, Hospital del Mar, Barcelona, Spain; Red SAMID (RD12/0026/0003), FEDER, Instituto de Salud Carlos III, Madrid, Spain \\ ${ }^{2}$ Childhood and Environment Research Group (GRIE), Institute Hospital del Mar Medical Research (IMIM), Barcelona, Spain \\ ${ }^{3}$ Cardiology Unit, Hospital del Mar, Barcelona, Spain \\ *Corresponding author: Oscar Garcia-Algar, Paediatrics Unit, Hospital del Mar, Barcelona, Spain; Tel: +34932483551; Fax: +34932483254; E-mail: \\ 90458@hospitaldelmar.cat
}

Received date: April 12, 2015; Accepted date: May 7, 2015; Published date: May 15, 2015

Copyright: @2015 Torres F. This is an open-access article distributed under the terms of the Creative Commons Attribution License, which permits unrestricted use, distribution, and reproduction in any medium, provided the original author and source are credited.

\begin{abstract}
Prolongation of the QT interval is the cause of about $10 \%$ cases of sudden infant death syndrome (SIDS) in newborns but it is also responsible for about $5 \%$ of sudden deaths in adults. LQTS is associated with life threatening arrhythmias such us torsade de pointes ventricular tachycardia or ventricular fibrillation (VF) leading to sudden death.

Congenital causes (channelopathies) of LQTS are associated to mutations with different hereditary pattern according affected gene but it has been proven that several drugs and electrolytic alterations can significantly increase the QT interval. However, the cost-effectiveness of neonatal electrocardiographic (EKG) screening has been questioned and despite studies made in newborns there is still no consensus about the best moment to make and electrocardiographic screening.

Previous studies demonstrate that newborns with QTc values over 470 msec often normalize by themselves in the first month of life. However in some cases these values remain high after first months of life. These cases are more frequently in determinate ethnics (Maghreb, Morocco and India-Pakistan). These races could be linked to cardiac electrical alterations with a hereditary pattern of variable penetrance. An EKG performed in the first month of life will allow the early identification of still asymptomatic infants with LQTS and also infants with some correctable congenital heart defects (CHD) not recognized by routine neonatal examinations. Appropriate therapy will prevent unnecessary deaths in infants, children, and young adults. At the light of this literature review we could assess that neonatal electrocardiographic screening is highly cost-effective and it may permit the early identification of infants at risk for SIDS.
\end{abstract}

Keywords: Newborn; EKG; Long QT syndrome; Sudden infant death syndrome; Channelopathies

\section{Introduction}

Long QT syndrome (LQTS) is an electrophysiological disorder characterized by a lengthening of the repolarization phase of the ventricular action potential (prolongation of the QT interval on the basis of a surface EKG). Patients with this condition tend to develop severe arrhythmias such as ventricular tachycardia, especially polymorphic ventricular tachycardia (VT) or torsade de pointes (TdP). These arrhythmias may lead to syncope, cardiac arrest or sudden cardiac death in infants but also in adults [1].

Prolongation of the QT interval is the cause of about $10 \%$ cases of sudden infant death syndrome (SIDS) in newborns, [2] furthermore it is responsible for approximately a $5 \%$ of sudden deaths in adults. (3) SIDS remains the leading cause of mortality in the first year of life in the postneonatal period. Despite many hypotheses, which mainly focus on respiratory or cardiac abnormalities the underlying mechanisms remain unclear. Among preventable causes of SIDS are the life-threatening arrhythmias caused by long-QT syndrome [3]. In $12 \%$ of patients with LQTS, sudden death was the first manifestation of the disease and in $4 \%$ this happened in the first year of life $[4,5]$.
Long QT syndrome (LQTS) is produced by alterations in genes that encode different ion channels (mainly potassium and sodium channels) that lead to potentially malignant cardiac arrhythmias. They are included among the so-called channelopathies, and genetic studies have enabled us to identify them as entities in their own right. Some of them may be suspected on the basis of the surface electrocardiogram (EKG), although, given the differences in penetrance, there are patients with these disorders and high risk of sudden death in whom they may be masked. The channelopathies most easily recognized by means of EKG, in addition to LQTS, are Brugada syndrome (BS), which affects the sodium channel, and the short QT syndrome (SQTS), which mainly affects the potassium channel [6]. LQTS can be congenital or acquired disease. In congenital cases, LQTS is a genetic disorder caused by mutations in several genes that mostly encode cardiac ion channels. The QT interval prolongation on the EKG promotes lethal arrhythmias during sympathetic activation, rest, or sleep, according to the specific genotype [2]. The congenital causes (channelopathies) are associated to mutations with different hereditary pattern according affected genes. The three most common genes associated with LQTS are KCNQ1, KCNH2, SCN5A (LQT1, LQT2 and LQT3 respectively). Types 1 and 2 are the most common forms (42 and $45 \%$ of genotyped families affected) and are the result of underactive potassium channels. Type 3 accounts for about $7 \%$ of genotyped families, and is the result of an overactive sodium channel. 
Risk of sudden death in types 1 and 2 is reduced with beta blocker medication [7]. It has been seen that there are 4 other genes less commonly associated such us KCNE1, KCNE2, KCNJ2 and CAV3. In the study made by Arnestad et al., they found a high prevalence of SCN5A mutations in SIDS which fits with their established role in causing arrhythmias during sleep, when most SIDS deaths occur [2]. Other mutations within the same sodium channel encoding gene (SCN5A) can result in under activity of the sodium channel and cause Brugada syndrome (death in this syndrome also occur during sleep due to ventricular fibrillation) and, although the QT interval is normal, there are other electrocardiography alterations (characterized by a coved-type ST-segment elevation $\geq 2 \mathrm{~mm}$ in more than one right precordial lead (V1-V3), followed by negative T waves) [8]. Recently, the first non-cardiac channel subtype of LQTS has been established for the previously elusive chromosome 4-linked LQT4, namely mutations in ankyrin B [9].

On the other hand, acquired LQTS, can be caused by the effect of several drugs, [10] electrolytic alterations (hypocalcaemia, hypomagnesaemia) and physiological influences like hypothermia or several brain injury [4]. However and despite all these studies made in newborns, there is no consensus about the best moment to make electrocardiographic screening. The aim of our review is to update the information about the effectiveness of a surface EKG as a screening method for LQTS.

\section{Methods}

A review has been made in PubMed with key words like "long QT syndrome" "sudden infant death syndrome" "neonatal electrocardiography" "channelopathies". We used well designed articles between 1992 and 2014 about LQTS and EKG screening recommendations. At the end we finally included a total number of 20 articles.

\section{Results}

The major changes in the paediatric EKG occur in the first year of life with the majority of normal adult values being abnormal in the newborn. Likewise, many normal newborn values and patterns would be abnormal in the adult. Identification of EKG abnormalities in the newborn can be the first step toward a meaningful act of preventive medicine in infants and adulthood. [5] It is established that newborns with prolonged QTc $(>440 \mathrm{msec})$ on the fourth day of life have an increased risk for sudden death [11]. The QT duration may change over time so it is recommended repeating EKG in those infants with a prolonged QTc on the first EKG. To decrease the rate of falsepositives, implementation of EKG screening at days 15 to 25 was advised [12]. The more prolonged the QTc interval, the greater the likelihood of its clinical significance. A QTc close to $500 \mathrm{msec}$ implies a clear abnormality even taking into account potential measurement errors [5]. The 18-year prospective study made by Schwartz et al., with EKGs recorded in $>33.000$ infants provided the first evidence that a prolonged QT interval on days 3 to 4 of life was associated with a significantly higher risk of Sudden infant death syndrome (SIDS) In this study they established a cutoff point for a normal QT interval corrected for heart rate (QTc) at $440 \mathrm{msec}$, with a prevalence of 0.9 per 1000 newborns. With respect to BS, there are no data on Japanese infants, but the prevalence among adults is $146.2 / 100000$ population. Finally, the SQTS was identified recently, and its prevalence among newborn infants is also unknown [11].
Healthcare programs have been classified in five grades of recommendation, from A to E. Grade B programs cost less than \$ 20,000 per quality-adjusted lifeyear (QALY) and are rated as "highly cost-effective' [13]. A study made by Quaglini demonstrated that EKG performed in the first month of life is cost-effectiveness. In this study it has been analyzed the cost-effectiveness of a nationwide program of neonatal EKG screening. Their primary analysis focused on LQTS alone; a secondary analysis focused on the possibility of identifying some CHDs also. Incremental cost-effectiveness analysis for the primary analysis shows that with the screening program, the cost per year of life saved is very low: $€ 11,740$. The cost for saving one entire life of 70 years would be $€ 820,000$. These figures define 'highly costeffective' screening programs. The secondary analysis provides even more cost-effective results [14].

\section{Discussion}

Early identification of the long-QT syndrome is particularly attractive because medications, use of automatic implantable defibrillators, and modifications of activities have been associated with significant reduction in risk of sudden death [15].

Some European countries have begun to consider the possibility of introducing in their National Health Services the performance of an EKG during the first month of life in all newborns, as part of a cardiovascular screening program $[5,6,16]$.

EKG screening and subsequent molecular testing of all neonates with QTc $>440$ msec could probably identify the majority of LQTS cases, including the ones that will not die of SIDS, but who are still at risk of sudden death at a young age [17].

Schwartz and cols. comment on the cost effectiveness of neonatal EKG in Italy. Their study demonstrated that neonatal EKG screening is highly cost-effective and a significant number of lives can be saved for an objectively small cost [13,18]. Regarding post-mortem molecular studies 10 to $15 \%$ of SIDS causes are due to LQTS and the early treatment is life-saving, in neonates and their relatives. Because of this and considering that the cost per year-of-life saved is 6,860 euros and the cost to save one life (estimating 70 years) is 500,000 euros $[11,17]$, electrocardiographic screening is cost-effective.

In a study made by Martí-Almor et al., it has been demonstrated that newborns with QTc values over $470 \mathrm{msec}$ often normalize by themselves in the first month of life, calling this phenomenon "spurious QTc". However in some cases these values remain high after first month of life. These cases appear more frequently in determinate ethnic groups (Maghreb, Morocco India-Pakistan). These ethnic groups could be linked to cardiac electrical alterations with a hereditary pattern of variable penetrance. The ethnic differences observed probably reflect genetic differences, although they may not necessarily be associated with a disease $[6,19,20]$.

\section{Conclusion}

The fact that, especially in infancy, malignant LQTS is usually associated with marked QT prolongation should stimulate an objective assessment of the pros and cons of widespread neonatal EKG screening performance, as recommended by the Guidelines of the European Society of Cardiology, [21] between the third and fourth weeks of life when prevalence of spurious marked QT prolongations (QTc $470 \mathrm{msec}$ ) is minimal [5]. 
Disadvantages of this screening are: must be necessary clinical accuracy, physician resources, medicolegal responsibility and risk. Advantages are multiple: quick and cheap diagnostic method, nonaggressive and painless, and the most important, the potential to prevent a sudden death [22].

Newborns with a prolonged QTc interval in the first days of life with a normalization of the values after the first month can be also at risk of sudden death when they became adults, especially if they are treated with some drugs that prolong the QTc interval. This aspect is remarkable because can be the first manifestation of a low penetrance disorder that otherwise will never be seen until a sudden death happens later on during life [5].

Nationwide screening programs have been proposed as a costeffective means in Europe to identify LQTS and thereby prevent some SIDS deaths [13].

In the light of this data we could assess that neonatal electrocardiographic screening is highly cost-effective (also in difficult economical settings) and it may permit the early identification of infants at risk for SIDS.

\section{References}

1. Barra S, Agarwal S, Begley D, Providência R (2014) Post-acute management of the acquired long QT syndrome. Postgrad Med J 90: 348-358.

2. Arnestad M, Crotti L, Rognum TO, Insolia R, Pedrazzini M, et al. (2007) Prevalence of long-QT syndrome gene variants in sudden infant death syndrome. Circulation 115: 361-367.

3. Muñoz Castellano J (2004) Sindrome de QT largo y Torsade de Pointes. Emergencias 16: 85-92.

4. Schwartz PJ, Priori SG, Napolitano C (2000) The long QT syndrome. In Zipes DP, Jalife J, eds. Cardiac electrophysiology: from cell to bedside. Philadelphia: WB Saunders 597-615.

5. Schwartz PJ, Garson A Jr, Paul T, Stramba-Badiale M, Vetter VL, et al. (2002) Guidelines for the interpretation of the neonatal electrocardiogram. A task force of the European Society of Cardiology. Eur Heart J 23: 1329-1344.

6. Martí-Almor J, Berrueco R, García-Algar O, Mur A, Bazán V, et al. (2008) Intervalo QT en recién nacidos de diferente origen étnico: utilidad del cribado con ECG neonatal. Rev Esp Cardiol 61: 980-982.

7. Skinner JR (2005) Is there a relation between SIDS and long QT syndrome? Arch Dis Child 90: 445-449.
8. Benito B, Brugada J, Brugada R, Brugada P (2009) Brugada syndrome. Rev Esp Cardiol 62: 1297-1315.

9. Tester DJ, Ackerman MJ (2005) Sudden infant death syndrome: how significant are the cardiac channelopathies? Cardiovasc Res 67: 388-396.

10. CredibleMeds

11. Schwartz PJ, Stramba-Badiale M, Segantini A, Austoni P, Bosi G, et al. (1998) Prolongation of the QT interval and the sudden infant death syndrome. N Engl J Med 338: 1709-1714.

12. Zupancic JA, Triedman JK, Alexander M, Walsh EP, Richardson DK, et al. (2000) Cost-effectiveness and implications of newborn screening for prolongation of QT interval for the prevention of sudden infant death syndrome. J Pediatr 136: 481-489.

13. Laupacis A, Feeny D, Detsky AS, Tugwell PX (1992) How attractive does a new technology have to be to warrant adoption and utilization? Tentative guidelines for using clinical and economic evaluations. CMAJ 146: 473-481.

14. Quaglini S Rognoni C, Spazzolini C, Priori SG, Mannarino S, et al. (2006) Cost-effectiveness of neonatal ECG screening for the long QT syndrome. Eur Heart J 27: 1824-1832.

15. Mahle WT, Sable CA, Matherne PG, Gaynor JW, Gewitz MH; American Heart Association Congenital Heart Defects Committee of the Council on Cardiovascular Disease in the Young (2012) Key concepts in the evaluation of screening approaches for heart disease in children and adolescents: a science advisory from the American Heart Association. Circulation 125: 2796-2801.

16. Berul CI, Perry JC (2007) Contribution of long-QT syndrome genes to sudden infant death syndrome: is it time to consider newborn electrocardiographic screening? Circulation 115: 294-296.

17. van Langen IM, Wilde AA (2006) ECG screening of newborns to avoid sudden infant death syndrome? Neth Heart J 14: 43-45.

18. Schwartz PJ (2006) Pro: Newborn ECG screening to prevent sudden cardiac death. Heart Rhythm 3: 1353-1355.

19. Ackerman MJ, Splawski I, Makielski JC, Tester DJ, Will ML, et al. (2004) Spectrum and prevalence of cardiac sodium channel variants among black, white, Asian, and Hispanic individuals: Implications for arrhythmogenic susceptibility and Brugada/long QT syndrome genetic testing. Heart Rhythm 1: 600-601.

20. Chang RK, Lan YT, Silka MJ, Morrow H, Kwong A, et al. (2014) Genetic variants for long QT syndrome among infants and children from a statewide newborn hearing screening program cohort. J Pediatr 164: 590-595.e1-3.

21. Guidelines of the European Society of Cardiology 2015.

22. Call for heart checks on babies (2006) BBC News. 